\title{
Light-Weight Monocular 3D Display Unit Using Polypyrrole Linear Actuator
}

\author{
Yuuki Kodama and Kunio Sakamoto \\ Department of Intelligence and Informatics, Konan University \\ 8-9-1 Okamoto, Higashinada, Kobe 658-8501, Japan \\ kunio@konan-u.ac.jp
}

\begin{abstract}
The human vision system has visual functions for viewing 3D images with a correct depth. These functions are called accommodation, vergence and binocular stereopsis. Most 3D display system utilizes binocular stereopsis. We have developed a monocular 3D vision system with accommodation mechanism, which is useful function for perceiving depth. This vision unit needs an image shift optics for generating monocular parallax images. But conventional image shift mechanism is heavy because of its linear actuator. To improve this problem, we developed a light-weight 3D vision unit for presenting monocular stereoscopic images using a polypyrrole linear actuator.
\end{abstract}

Keywords: head mounted display, monocular stereoscopic display, real-time stereogram, 3-D display.

\section{Introduction}

A study of virtual-reality system has been popular and its technology has been applied to medical engineering, educational engineering, a CAD/CAM system and so on. The 3D imaging display system has two types in the presentation method; one is a 3D display system using a special glasses and the other is the monitor system requiring no special glasses. A display system with no special glasses is useful for a 3D TV monitor, but this system has demerit such that the size of a monitor restricts the visual field for displaying images. The 3D display system using special glasses can display virtual images over a wide area. The human vision system has visual functions for viewing $3 \mathrm{D}$ images with a correct depth. These functions are called accommodation, vergence and binocular stereopsis. Accommodation is a useful function for perceiving a depth by the monocular vision system. Binocular stereopsis is one of the most important processes for the human 3D perception. Then most 3D display system utilizes this binocular stereopsis. The binocular vision mostly utilizes next two functions. One is simultaneous perception, which is an ability to perceive dichoptically presented images simultaneously and in the correct position. The other is binocular fusion, which is an ability to perceive two dichoptic images in left and right eyes as one image. Meanwhile accommodation enables us to perceive a depth by the monocular vision system. And humans have the function called the simultaneous perception. Using this function, humans perceive dichoptically presented images 
simultaneously and in the correct position. So the authors have developed a monocular 3D vision system which enables us to provide correct and natural 3D images with a depth perceiving by the accommodation.

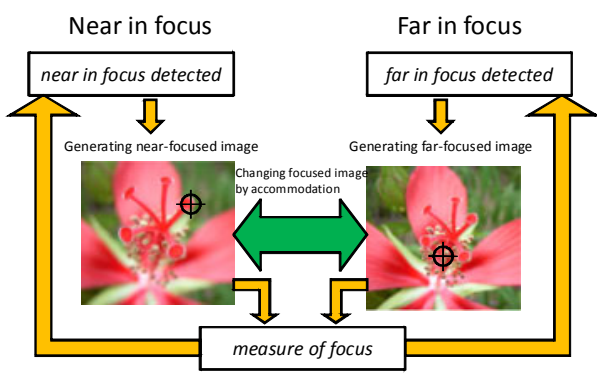

Fig. 1. Flow of generating focused image

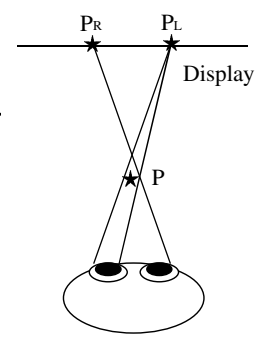

(a)

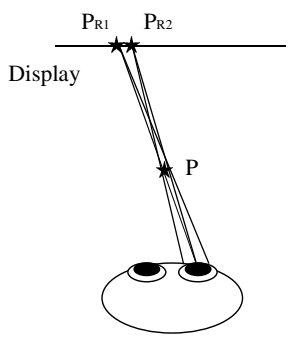

(b)

Fig. 2. The principle of a stereogram

\section{Monocular 3D Display}

To realize natural 3D viewing, we have developed the monocular vision system, which can directly project stereoscopic image on a retina, and a $3 \mathrm{D}$ image generation system, which can make 3D computer graphics in accordance with accommodation. Assume that an actual object is in the real world. When you perceive this object, a part of the projected image on a retina might be a blur by the lens of an eye. It is a physiological response called as accommodation. In case of virtual 3D image viewing as shown in fig. 1, you can watch a correct 3D image as the actual object is in there if the projected retina image has appropriate blur in compliance with focus adjustment of your eye. Then you might perceive virtual images with same accommodation as you watch real objects. Thus a monocular $3 \mathrm{D}$ vision system can provide correct $3 \mathrm{D}$ viewing with accommodation, vergence and binocular stereopsis and without a tired feeling at long time watching when the retina image is directly projected and external stimulation induces the focus adjustment by changing the thickness of an eye lens.

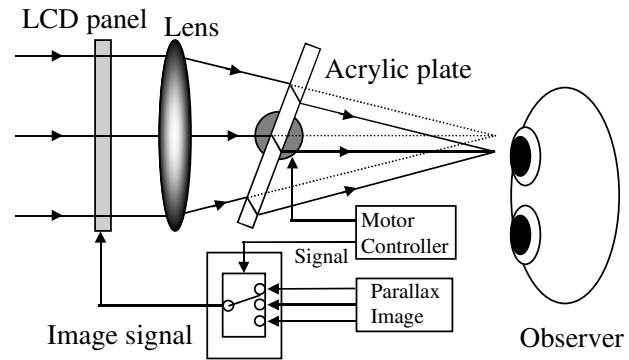

Signal controller

Fig. 3. Optical layout of monocular 3D display

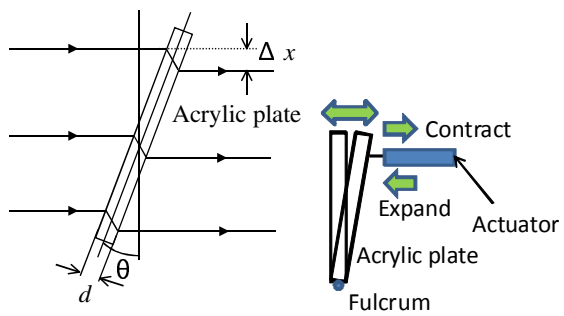

Fig. 4. Image shifting optics 
Fig. 2 shows the principle of a reconstruction of the 3D image by the stereoviewing. Fig. 2 (a) shows the optical configuration of a conventional stereogram. To display a point object $\mathrm{P}$, observers turn on a point $\mathrm{P}_{\mathrm{L}}$ for the left eye and a point $\mathrm{P}_{\mathrm{R}}$ for the right eye as shown in this figure. The observer perceives that a point object exists on a spatial position $\mathrm{P}$ due to the binocular parallax. Fig. 2 (b) shows the optical configuration of a monocular multi-view stereogram. To display a point object $\mathrm{P}$, observers turn on points $\mathrm{P}_{\mathrm{R} 1}$ and $\mathrm{P}_{\mathrm{R} 2}$ for the right eye as shown in this figure. At the monocular multi-viewing, the observer adjusts the focal length of an eye to match with the spatial position $\mathrm{P}$, then projected images of pixels $\mathrm{P}_{\mathrm{R} 1}$ and $\mathrm{P}_{\mathrm{R} 2}$ are focused to the same position on the retina. So the observer naturally perceives that a point object exists on a spatial position $\mathrm{P}$ due to the monocular parallax. Fig. 3 shows the principle of the 3D vision system using monocular stereoscopy. This display system consists of an LCD panel, an acrylic plate and an optical lens. The observers perceive parallax images at the just point, which the optical lens converges the light on. To perceive multiple parallax images with just one eye, the image shifting optics consists of a parallel plane acrylic plate, whose inclination causes the image to shift as shown in fig. 3. An LCD panel is used as the displaying plane of parallax images. The signal controller sends an image signal to the LCD panel to the tune of a control signal. Then the displaying plane of parallax images creates monocular multi-viewing images.

\section{Improvement of Image Shift Optics}

As shown in fig. 4, the image shift $\Delta x$ is generated by an inclined acrylic plate. To incline the plate, former vision unit used a motor. Therefore its weight is heavy so that observers wear it as glasses. To improve this problem, the authors developed new image shift mechanism for monocular 3D vision. This image shifting optics consists of an acrylic plate and a polypyrrole linear actuator as shown in fig. 4. Fig. 5 shows the structure of the polypyrrole actuator. This plastic film actuator is made of the polypyrrole. The thin layer of polypyrrole is attached on an acetate film as a support film. The polypyrrole can expand when it absorbs moisture in the air. When it discharges moisture, it can contract. Meanwhile the polypyrrole can also contract when the voltage is applied to it. The principle lies in the desorption of water vapor caused by Joule heating, where the electric field controls the sorption and the film absorbs and desorbs moisture reversibly in response to the applied voltage. The plastic film actuator is bent because the polypyrrole layer contacts but the acetate film cannot contract as shown in fig. 6 . The polypyrrole film containing perchlorate is electrochemically synthesized by the anodic oxidation of pyrrole. The plastic film actuator can incline the acrylic plate when this film actuator is bent (or contracts) by controlling the applied voltage as shown in fig. 4. The film actuator is thin and light. So the authors could develop the light-weight monocular display unit for $3 \mathrm{D}$ viewing with a correct and natural depth using this polypyrrole actuator. 


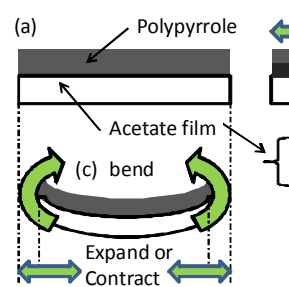

Fig. 5. Polypyrrole linear actuator

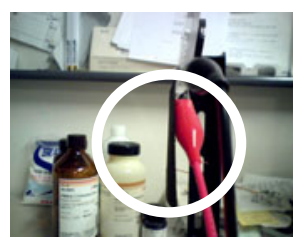

(a) off state

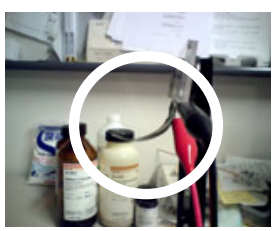

(b) on state

Fig. 6. Appearance of polypyrrole linear actuator

Acknowledgments. This research is partially supported by "Grant-in-Aid for Young Scientists(B)" \#20700112 and "Scientific Research (C) (General)" \#20500481 from Ministry of Education, Culture, Sports, Science and Technology Japan(MEXT). 